\title{
Thermo-Optical parameters of propylene carbonate as a laser dye solvent
}

\author{
Hassan A. El-Kashef, Asmaa. S. Rezk \\ Physics Department, Faculty of Science, Tanta University, Tanta, Egypt \\ Email address: \\ hassanelkashef51@yahoo.com (H. A. El-Kashef)
}

\section{To cite this article:}

Hassan A. El-Kashef, Asmaa. S. Rezk. Thermo-Optical Parameters of Propylene Carbonate as a Laser Dye Solvent. Optics. Vol. 2, No. 5, 2013, pp. 61-66. doi: 10.11648/j.optics.20130205.12

\begin{abstract}
The dual laser wavelength Mach-Zehnder interferometric technique is used to study the thermal and optical properties of propylene carbonate (PC), which consider as important polar dielectric solvent for many laser dyes. Using Argon and He-Ne laser at wavelengths 514.5 and $632.8 \mathrm{~nm}$ as a light sources and Mach-Zehnder interferometer, many thermal and optical parameters are measured and many others are calculated. The experimental technique is used for measuring the refractive index and its gradient with temperature. Applying Cauchy,s equation the dispersion of PC can be studied. Using Murphy-Alpert,s equation the gradient of refractive index $\mathrm{dn} / \mathrm{dT}$ as a function of wavelength and the density versus temperature are calculated and plotted. On the other hand, the constants A and B of Cauchy,s equation as a function of temperature were studied. Additionally, using Fresnel equations many optical properties such as transmittance and reflectance as a function of incident angle, temperature and wavelength are calculated and represented. This paper gives new data measured at high frequencies to be useful and available for all workers in the field of dye lasers (designers and researchers).
\end{abstract}

Keywords: Laser Interferometry, Laser Dye Solvents, Thermo-Optical Properties

\section{Introduction}

Dye laser has unique characteristics that govern and limit our ability to control it. In previous literature the general properties and specific issues associated with dye lasers were discussed [1]. The gain medium of the dye lasers, the organic dye concentration in the solvent typically ranges from $10^{-2}$ to $10^{-5}$. One of the chief attributes the interaction between the dye solvent can effect the wavelength and efficiency of the optical gain [2]. The solvent fulfills also several functions during a chemical reaction:

It solvates the reactions and reagents so that they dissolve this facilitates collisions between the reactants and reagents that must occur in order to transform the reactants to products.

The solvent provides a means of temperature control.

It increase the energy of the colliding particles so that they will react more quickly.

It absorbs heat that is generated during an exothermic reaction.

Different research were published for measuring the molecular properties of nonpolar laser dye solvents, see for example Ref [3]. other published paper studied the wavelength and temperature dependence properties of human blood serum. The paper gives the properties of a biological sample (serum) which cover about $70 \%$ of our body weight. The achieved results is consider as a guide for using laser as a treatment tool in clinical studies [4]. On an operational basis, the selection of an appropriate solvent for a certain dye is guided by theory and experience. Some of he physical properties of solvents are already reported in literature, which were measured using an electrical method at low frequency range, see for example Ref. [5]. In fact the laser dye solvents are used at high frequencies (UV, visible and IR), therefore it is of interest to study its properties at these high frequencies.

Because of the many different applications of PC as familiar polar dielectric solvent for many laser dyes such as: PYRIDINE $\quad 1 \quad\left(\mathrm{C}_{19} \mathrm{H}_{23} \mathrm{~N}_{2} \mathrm{O}_{4} \mathrm{Cl}\right), \quad$ OXAZINE 750 $\left(\mathrm{C}_{14} \mathrm{H}_{24} \mathrm{~N}_{2} \mathrm{O}_{5} \mathrm{Cl}\right)$, STYRYL $8\left(\mathrm{C}_{21} \mathrm{H}_{23} \mathrm{~N}_{2} \mathrm{SO}_{4} \mathrm{Cl}\right)$, STYRYL $9 \mathrm{M}\left(\mathrm{C}_{27} \mathrm{H}_{31} \mathrm{~N}_{2} \mathrm{O}_{4} \mathrm{SCl}\right)$, STYRYL $11\left(\mathrm{C}_{23} \mathrm{H}_{25} \mathrm{~N}_{2} \mathrm{O}_{4} \mathrm{Cl}\right)$, and DNTTCI $\left(\mathrm{C}_{33} \mathrm{H}_{29} \mathrm{~N}_{2} \mathrm{~S}_{2} \mathrm{I}\right)$, we are concerned with the studies of its thermal and optical properties. The present measurements depends on accurate determinations of refractive index and its thermal coefficient at laser wavelengths 514.5 and $632.8 \mathrm{~nm}$ using a dual laser Mach- 
Zehnder interferometric technique. From the experimental results, one can determine the optical dispersion $\mathrm{dn} / \mathrm{d} \lambda$, the thermal coefficient of refractive index $\mathrm{dn} / \mathrm{dT}$ and $\mathrm{dn} / \mathrm{d} \lambda$ as a function of wavelength $\lambda$ and the Cauchy's constants A and $\mathrm{B}$ versus temperature. Additionally, using a derived relationship between the refractive index and density we can calculate and plot the density as a function of temperature at optical frequencies. On the other hand, the reflectance and transmittance at different physical variables (angle of incidence, temperature and wavelength) are calculated and plotted.

Table 1. The refractive index $n, d n / d T$ at $514.5 \mathrm{~nm}, 632.8 \mathrm{~nm}$ and $d n / d \lambda$ at $600 \mathrm{~nm}$

\begin{tabular}{ccc}
\hline constants & $\boldsymbol{\lambda}=\mathbf{5 1 4 . 5} \mathbf{~ n m}$ & $\boldsymbol{\lambda}=\mathbf{6 3 2 . 8} \mathbf{~ n m}$ \\
\hline $\mathrm{n}$ & 1.45202 & 1.41924 \\
$\mathrm{dn} / \mathrm{dT}$ & $-17.6 \times 10^{-5}$ & $-16.3 \times 10^{-5}$ \\
$\mathrm{dn} / \mathrm{d} \lambda$ at $600 \mathrm{~nm}$ & $2.3704 \times 10^{-4}$ & \\
\hline
\end{tabular}

\section{Theoretical}

Using a certain physical parameters (refractive index and its variation with temperature), one can obtain quantitative information about structure parameters of PC. The following experimental linear relationship of MachZehnder interferometer as described in [6] is used to determine the refractive index:

$$
1 / \sin ^{2} \theta / 2=\{2 \mathrm{~d}(\mathrm{n}-1) / \mathrm{n} \lambda\} \cdot 1 / \mathrm{N}+2 / \mathrm{n}
$$

where $\theta$ is the angle of incidence, $\mathrm{n}$ is the refractive index, $\mathrm{d}$ is the sample thickness, $\lambda$ is the laser wavelength and $\mathrm{N}$ is the number of fringes that shifted from the field of view.

From the slope of graphical representation $1 / \sin 2 \theta / 2$ on the $\mathrm{y}$-axis and $1 / \mathrm{N}$ on $\mathrm{x}$-axis we can determine $\mathrm{n}$ and $\mathrm{d}$. The thermal coefficient of refractive index $\mathrm{dn} / \mathrm{dT}$ was determined by measuring $\mathrm{n}$ at different temperature using the following formula:

$$
\mathrm{n}_{\mathrm{T} 1}-\mathrm{n}_{\mathrm{T} 2}=\Delta \mathrm{N} \cdot \lambda / \mathrm{d}
$$

Equation (2) is obtained from equation (1), when $\theta=0$ and $\mathrm{n}_{\mathrm{T} 1}, \mathrm{n}_{\mathrm{T} 2}$ are the refractive indices at two different temperatures $\mathrm{T}_{1}, \mathrm{~T}_{2}$.

The refractive properties can be extracted by applying Cauchy's equation:

$$
\mathrm{n}_{\lambda}=\mathrm{A}+\mathrm{B} / \lambda^{2}
$$

where $\mathrm{A}$ and $\mathrm{B}$ are constants at a given concentration and temperature.

Substituting the determined values of refractive indices at the two wavelengths (514.5 and $632.8 \mathrm{~nm}$ ) in equation (3), the constants A and B can be calculated by solving the equations, and the refractive indices at different wavelengths can be calculated and accordingly the dispersion curve is plotted.

Important refractive parameters for PC are also studied. The dependence of $-\mathrm{dn} / \mathrm{dT}$ as a function of wavelength is calculated using the Murphy and Albert,s relationship [7]:

$$
\mathrm{dn} / \mathrm{dT}=-3 \mathrm{n}\left(\mathrm{n}^{2}-1\right) \gamma / 2\left(2 \mathrm{n}^{2}+1\right)
$$

At a given wavelength and temperature, the differentiation of Lornetz-Lorenz equation (4) in Ref. (8) gives the following formula:

$$
\rho=\left(n^{2}-1\right)\left(n^{2}+2\right) / 6 n(d n / d \rho)
$$

If the refractive index and density $\rho$ at a certain wavelength are known, the value of $\mathrm{dn} / \mathrm{d} \rho$ can be calculated from equation (5). This leads to the evaluation of density as a function of temperature.

On the other hand, The transmission coefficient (transmittance) and reflectance is calculated against the angle of incidence using the following Fresnel equations for parallel and perpendicular polarized components [9]:

$$
\begin{gathered}
\mathrm{t}_{\|}=\sin 2 \theta \sin 2 \varphi / \sin ^{2}(\theta+\varphi) \cos ^{2}((\theta-\varphi) \\
\mathrm{t}_{\perp}=\sin 2 \theta \sin 2 \varphi / \sin ^{2}(\theta+\varphi)
\end{gathered}
$$

$t_{\|}$and $t \perp$ are the parallel and perpendicular polarized components of the transmittance and $\varphi$ is the angle of refraction.

The dependence of wave field reflection on the angle of incidence $(\theta)$ is calculated using the following equations [9]

$$
\begin{aligned}
& r_{\|}=\tan ^{2}(\theta-\theta) / \tan ^{2}(\theta+\varphi) \\
& r_{\perp}=\sin ^{2}(\theta-\varphi) / \sin ^{2}(\theta+\varphi)
\end{aligned}
$$

The values of refractive indices as a function of temperature are substituted in the following Fresnel formula for normal incidence $\left(r_{\|}=r \perp=r\right)$ to calculate the reflectance $r$ as a function of a temperature [9]:

$$
\mathrm{r}=\{(\mathrm{n}-1) /(\mathrm{n}+1)\}^{2}
$$

Using the equation:

$$
\mathrm{t}=4 \mathrm{n} /(\mathrm{n}+1)^{2}
$$

The transmittance ( $\mathrm{t}$ ) as a function of wavelength and temperature is calculated and plotted.

Table 2. The density as a function of temperature

\begin{tabular}{cc}
\hline $\mathbf{T}^{\mathbf{}} \mathbf{C}$ & Density $\boldsymbol{\rho}$ at $\boldsymbol{\lambda}=\mathbf{6 3 2 . 8} \mathbf{~ n m}$ \\
\hline 20 & 1.1890 \\
25 & 1.1863 \\
30 & 1.1835 \\
35 & 1.1809 \\
40 & 1.1782 \\
45 & 1.1755 \\
50 & 1.1728 \\
\hline
\end{tabular}

\section{Experimental}

The schematic diagram of the experimental set-up as in Fig. (1) is used for measuring the refractive index and its thermal coefficient is the same as that described intensively 
in Ref. $[4,8,10]$. An Argon laser beam (Cyonics C2213150ML, $\lambda=514.5 \mathrm{~nm}$ ) and a He-Ne laser beam (Spectra Physics $155, \lambda=632.8 \mathrm{~nm}$ ) are separately used to illuminate a Mach-Zehnder interferometer. A cell of fresh sample of PC (purity of $99.5 \%$ ) is inserted on one of its arms where the angle of incident light can be adjusted using a calibrated rotating table. By changing the angle of incidence and counting the number of fringes shifted from the field of view, one can calculate the refractive index from the graphical representation of Eq. (1) at the two different wavelengths as given in Fig. (2). The thermal coefficient of refractive index can be determined using Eq. (2). This accomplished by introducing the sample cell in a temperature control unit at normal incidence, changing the temperature and recording the number of shifted fringes. The negative thermal coefficient of refractive index can be determined from the slope of the relationship between the refractive index and temperature as represented in Fig. (3). The accuracy measurement of the experimental technique are investigated and explained as given in Ref [3].

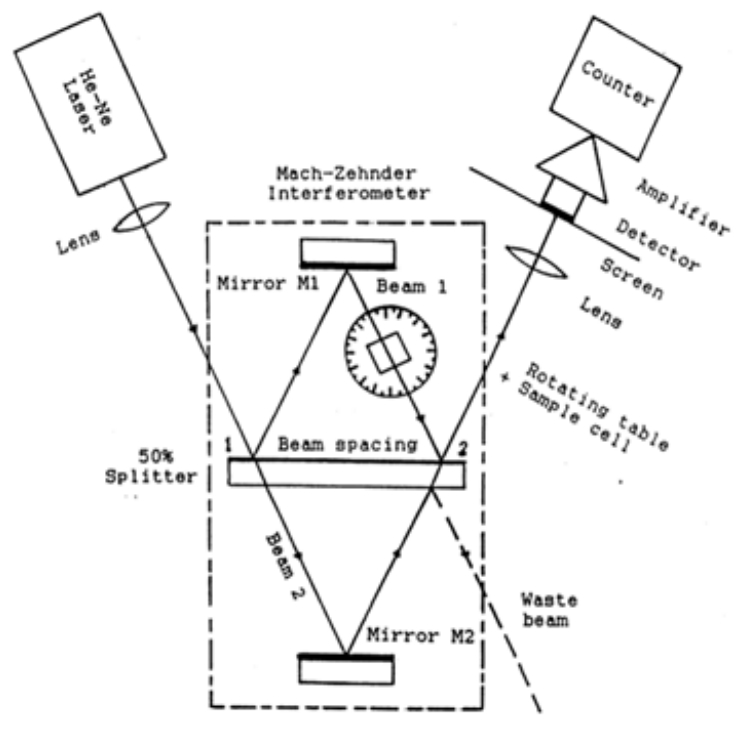

Figure (1). Schematic diagram of the Experimental set up.

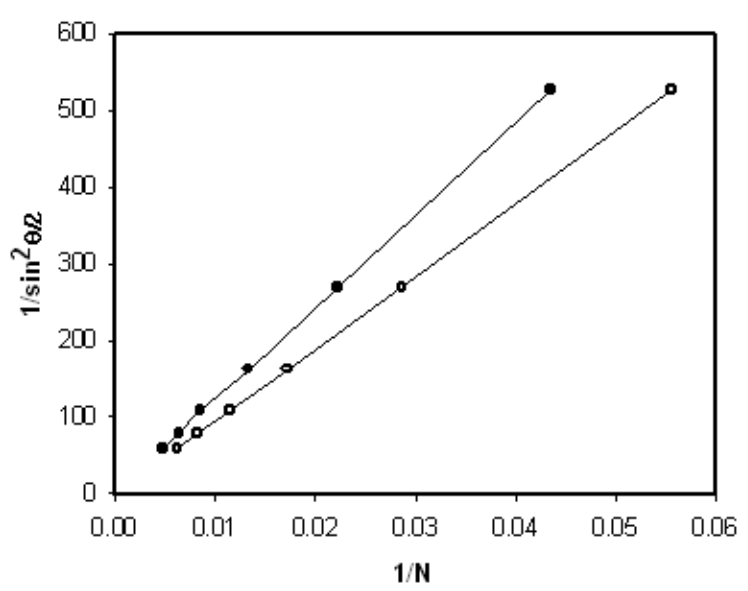

Figure (2). representation of Eq. (1) for refractive index determination.



Figure (3). refractive index as a function of temperature.

\section{Refractive Parameters}

Using the measured refractive indices at two different wavelengths as extracted from Fig (2) $(n=1.41924$ at $\lambda=$ $632.8 \mathrm{~nm}$ and $\mathrm{n}=1.45202$ at $\lambda=514.5 \mathrm{~nm}$ ), we can solve Eq. (3) to calculate Cauchy's Constants A and B as follows :

$$
\mathrm{n}=1.35531+25600.645 / \lambda^{2}
$$

where $\mathrm{A}=1.35531$ and $\mathrm{B}=25600.645$. From Eq. (10) the refractive indices at different wavelengths are calculated and the optical dispersion of PC can be represented as shown in Fig. (4). Differentiating Eq. (3) with respect to $\lambda$ $\left(\mathrm{dn} / \mathrm{d} \lambda=-2 \mathrm{~B} / \lambda^{3}\right)$, the optical dispersion $\mathrm{dn} / \mathrm{d} \lambda$ is calculated as a function of $\lambda$ and represented as in Fig. (5). The temperature gradient $\mathrm{dn} / \mathrm{dT}$ at different wavelengths are calculated using Eq.(4). Applying $\lambda=632.8 \mathrm{~nm}$ and $\mathrm{dn} / \mathrm{dT}$ $=-16.3 \times 10^{-5}$, we can determine the unknown $\gamma$ to be as in the following:

$$
\mathrm{dn} / \mathrm{dT}=-3.7961 \times 10^{-4}\left\{3 \mathrm{n}\left(\mathrm{n}^{2}-1\right)\right\} /\left\{2\left(2 \mathrm{n}^{2}+1\right\}\right.
$$

Substituting the values of $\mathrm{n}$ at different wavelengths in Eq. (11) one can represent $\mathrm{dn} / \mathrm{dT}$ versus $\lambda$ as given in Fig. (6). Table (1) gives the values of $n, d n / d T$ at two different wavelengths and $\mathrm{dn} / \mathrm{d} \lambda$ at the middle of visible range 600 nm.

As long as the refractive index and density at a certain wavelength are known the value of $\mathrm{dn} / \mathrm{d} \rho$ of Eq. (5) can be calculated to be in the form:

$$
\rho=2.48681\left(n^{2}-1\right)\left(n^{2}+2\right) / 6 n
$$

where $\mathrm{dn} / \mathrm{d} \rho=0.402121$. This leads to the calculation of density as a function of temperature as given in table (2). On the other hand, one can solve Cauchy's equation to calculate the constants $\mathrm{A}$ and $\mathrm{B}$ as a function of temperature as given in Figs. 7(a,b). The calculation of $A$ and $B$ are carried out as long as the refractive indices at two different wavelengths are known at a certain temperature. 


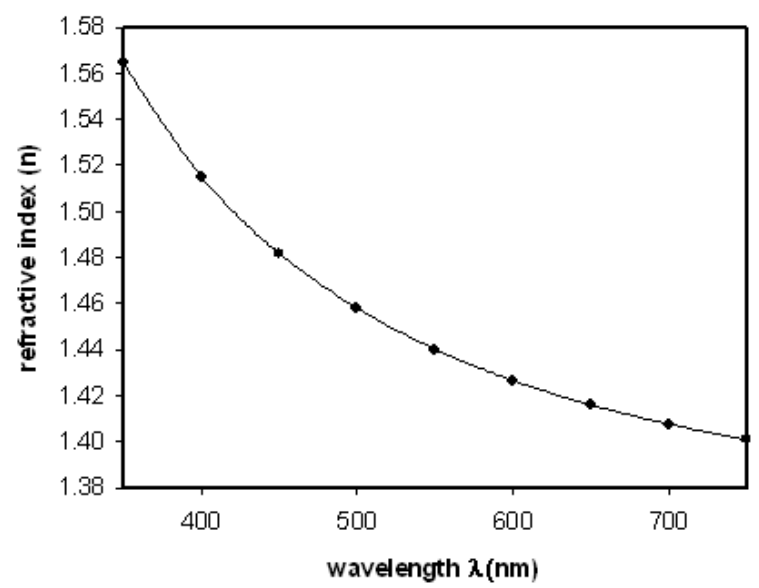

Figure (4). the optical dispersion of $P C$.

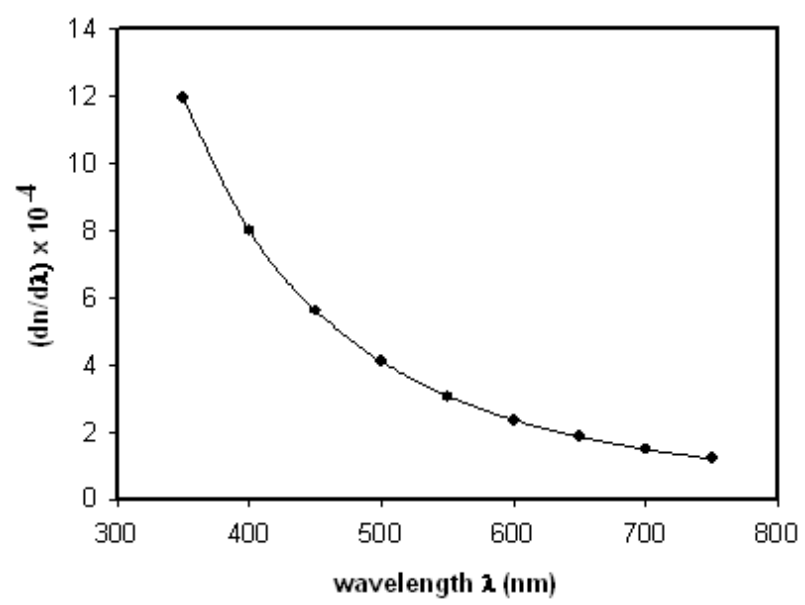

Figure (5). dn/d $\lambda$ as a function of wavelength $\lambda$.



Figure (6). $d n / d T$ versus wavelength $\lambda$.

\section{Optical Parameters}

Different phenomena take place when light hits a given medium. The optical constants of PC, such as reflectance, refraction, absorption, spread and transmittance are very important in the field of dye laser. The interrelationship between these phenomena determines the distribution of laser light in the media. Therefore, we are interested to determine these constants for PC using the measured data of refractive indices at the same two laser wavelengths.

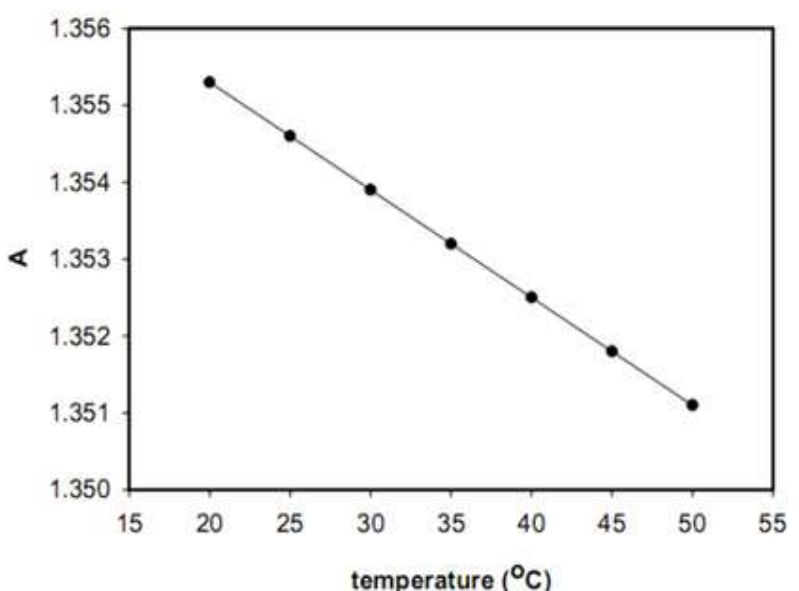

(a)

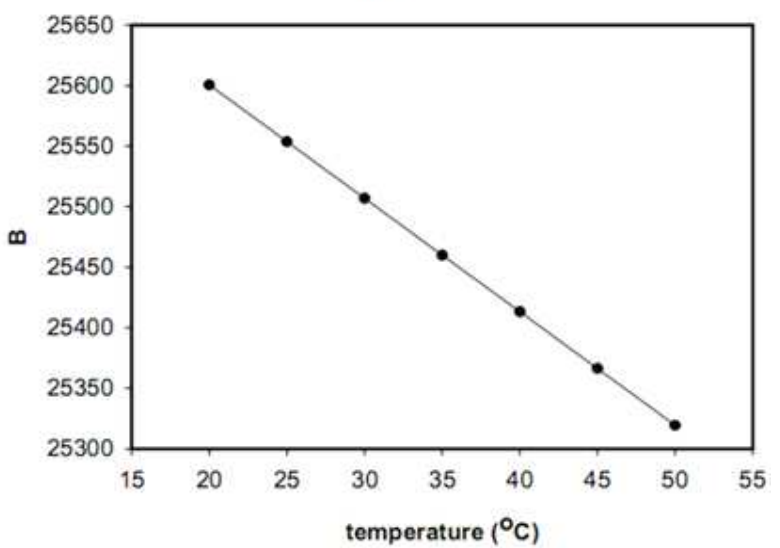

(b)

Figure $7(\boldsymbol{a}, \boldsymbol{b})$. Cauchy's constants as a function of temperature.

By applying Fresnel formulas, the dependence of wave field transmittance on the incidence angle $\theta$ is calculated according to Eqs. 6, 7 and represented as shown in Fig. (8). On the other hand, the reflection coefficient (reflectance) is calculated against the angle of incidence according to the Fresnel equations $(8,9)$ for parallel and perpendicular polarized components and plotted as in Fig.(9) .

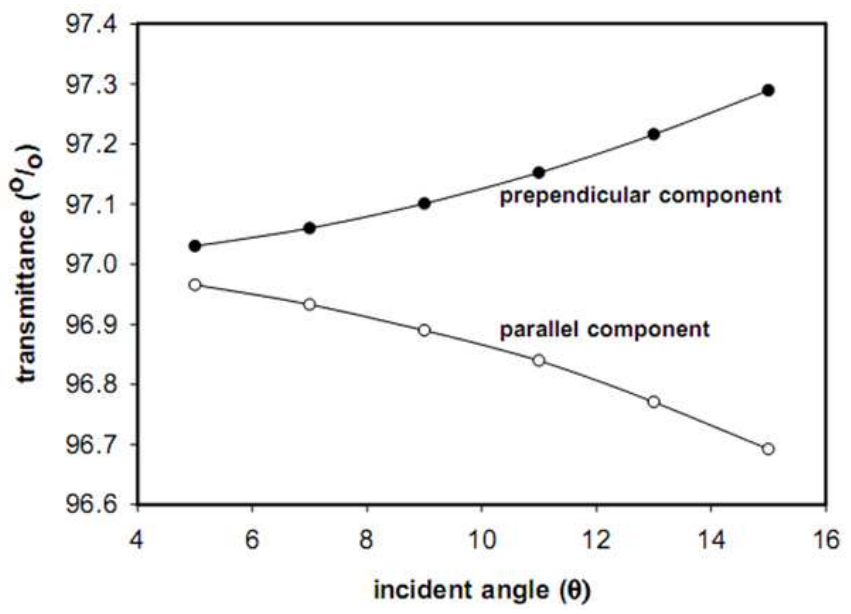

Figure (8). transmittance \% versus the angle of incidence. 


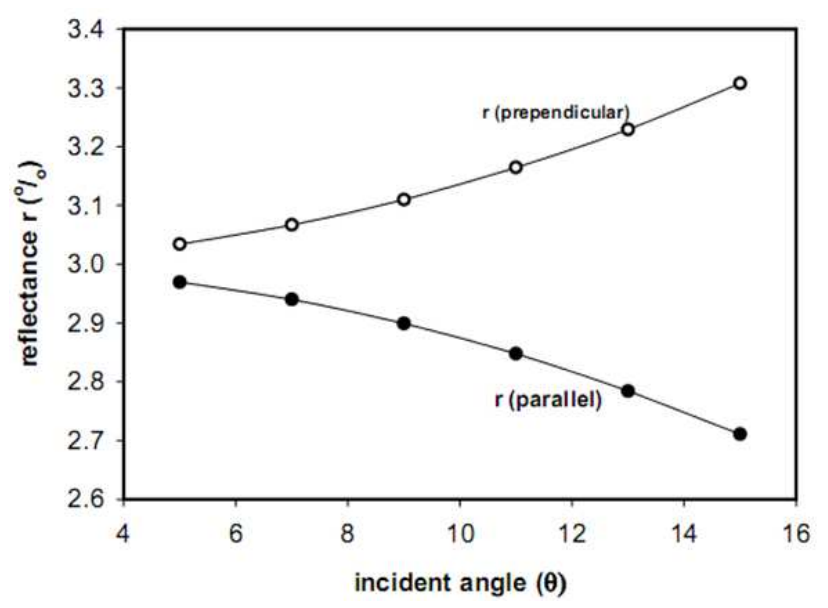

Figure (9). reflectance \% versus the angle of incidence

As long As the refractive indices at different temperatures are known, we can apply Fresnel formula for normal incidence $\left(r_{\|}=r_{\perp}=r\right)$. Accordinglly, Eqs. $(10,11)$ are used to calculate the reflectance and transmittance as a function of temperatures. Figs. $(10,11)$ shows the representation of the relationships. Additionally, the reflectance and transmittance as a function of wavelength are plotted as given in Figs. $(12,13)$.



Figure (10). reflectance versus temperature .

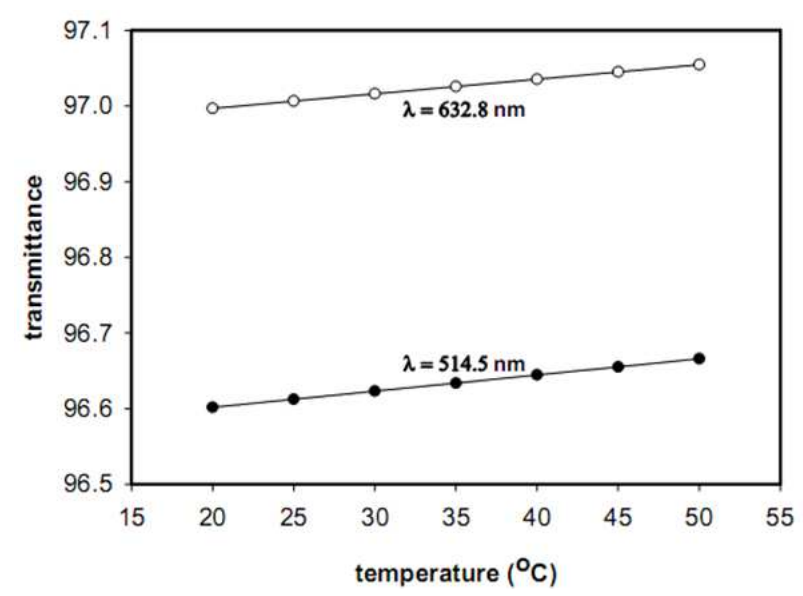

Figure (11). transmittance versus temperature .



Figure (12). reflectance versus wavelength .

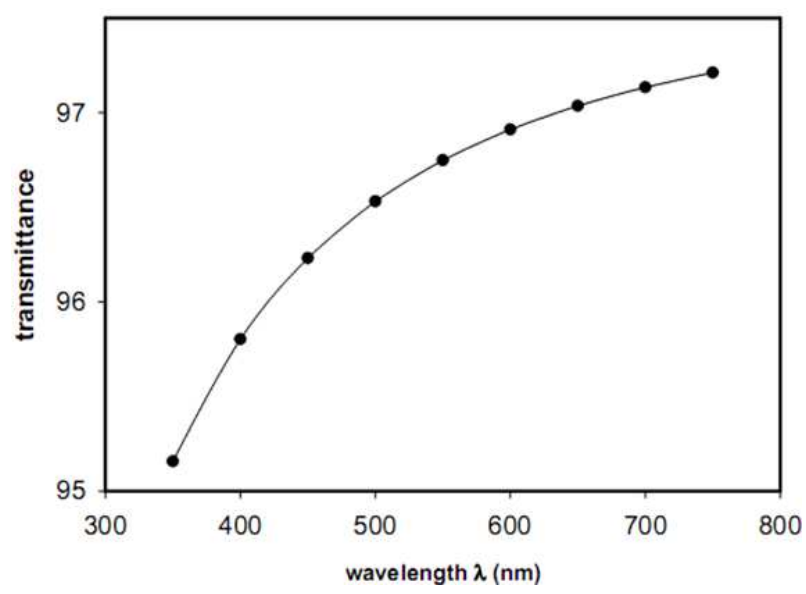

Figure (13). transmittance versus wavelength .

In conclusion, for the importance of PC as laser dye solvents and many other applications, its thermo-optical properties are investigated using dual laser Mach-Zehnder interferometric technique. The determined values of refractive indices and its gradient with temperatures leads to the study of different refractive and optical properties which play a major role in the frequency selection and stability of dye laser. Between these properties, The variation of refractive index with temperature, the optical dispersion, The variation of $\mathrm{dn} / \mathrm{dT}$ and $\mathrm{dn} / \mathrm{d} \lambda$ as a function of wavelength, the dependence of Cauchy's constants A and $\mathrm{B}$ as a function of temperature, On the other hand, the wave field reflectance and transmittance as a function of incident angle, temperature and wavelength are calculated and represented. Additionally, using a derived equation, the density of $\mathrm{PC}$ as a function of temperatures are calculated and tabulated.. The obtained data reflect the genereal properties in relation to the temperature, wavelength and the angle of incidence. This means a lot of useful information data are studied which could be of scientific interest for dye laser users, designers and many other applications. .The paper gives a lot of novel data for PC. These data are measured at high frequencies to be consider as a reference for the designers and researchers in the field of dye lasers. 


\section{References}

[1] F. J. Duarte et al., Dye Lasers Principles, Academic Press, San Diego 1990.

[2] Wellegehausen, L. Laepple, H. Welling, Applied Phys. 6 (1975) 335 .

[3] Hassan El-Kashef, J. Modern. Opt. 46 (1999) 1389.

[4] H. El-Kashef et al, Opt. \& Laser Technol, 31(1999)181.
[5] Lide DR, Frederikse HPR. CRC Handbook of Chemistry and Physics, $76^{\text {th }}$ ed. Boca Raton: CRC,1995-1996.

[6] H. El-kashef et al. , Applied Opt. 33 (1994)3540.

[7] C. G. Murphy, S. S. Alpert, Am. J. Phys. 39 (1971) 834

[8] Hassan El-Kashef, Opt. Mater. 8 (1997) 175

[9] Born. M., Wolf E., Principles of optics, $4^{\text {th }}$ ed. New York: Mc Graw-Hill, 1979.

[10] H. El-Kashef, Rev. Sci. Instrum. 65 (1994) 2056. 